\title{
Efectos de algunos parámetros de reacción en la producción de ésteres etílicos empleando una combi-lipasa de TLL y CALB en Lewatit VP OC 1600
}

\author{
Esteban C. Toro; César A. Godoy* \\ Departamento de Química (GIPAB-LIBB). Universidad del Valle, Calle 13 \# 100 - 00, Cali, Colombia. \\ *cesar.godoy@correounivalle.edu.co
}

Fecha recepción: diciembre 2 de 2019

Fecha aceptación: mayo 8 de 2020

\begin{abstract}
Resumen
La optimización de mezclas de lipasas inmovilizadas (combi-lipasas) ha sido aplicada como estrategia para incrementar la velocidad y el rendimiento en la producción de ésteres alquílicos de ácidos grasos o en aceites vegetales hidrolizados frente a la biocatálisis convencional (catalizadores monoenzimáticos). Previamente, se identificó la Combi-lipasa (CL) de Thermomyces lanuginosus (TLL) y Candida antarctica isoforma B (CALB) en la proporción 75:25 en el soporte Lewatit VPOC 1600 (LW) como la más activa con oleína de palma, de acuerdo a la cantidad de proteína inmovilizada y su eficiencia en la producción de ésteres etílicos (EE) en condiciones suaves de reacción. Sin embargo, su estabilidad en diferentes condiciones no ha sido estudiada. Así, se determinó que esta CL produce EE de modo estable bajo diferentes temperaturas y ante la presencia de agua y ácidos grasos libres, por contraste, la adición de glicerol generó disminuciones de hasta el $70 \%$ en la síntesis de ésteres etílicos. Aplicando el modelo de Arrhenius para una reacción de pseudoprimer orden, se determinó una Ea de 21,4 $\pm 1,4 \mathrm{~kJ} / \mathrm{mol}$, significando una disminución del $5 \%$ frente a su componente principal (TLL-LW). Adicionalmente, el uso de un aceite diferente (de soya) generó un aumento del $20 \%$ en la producción de EE. Por último, se realizó un estudio de docking y la simulación cinética del sistema usando un modelo desarrollado previamente, pero no se logró una clara correlación con los resultados experimentales. Al margen de esto, por su robustez, la CL estudiada presenta un alto potencial de aplicación en síntesis de ésteres etílicos.
\end{abstract}

Palabras clave: Biocatalizadores; Combi-lipasas; Oleína de palma; Docking; Ésteres Etílicos; Enzimas. 


\title{
Effects of some reaction parameters on the production of ethyl esters using a combi-lipase of TLL and CALB in Lewatit VP OC 1600
}

\begin{abstract}
Optimization of mixtures of immobilized lipases (combi-lipases) has been applied as a strategy to increase the rate and the yield in the production of alkyl esters of fatty acids or in hydrolyzed vegetable oils compared to conventional biocatalysis (monoenzymatic catalysts). Previously, Combi-lipase (CL) of Thermomyces lanuginosus (TLL) and Candida antarctica isoform B (CALB) in the proportions 75:25 in the Lewatit VPOC 1600 (LW) support were identified as the most active with palm olein, from according to the amount of immobilized protein and its efficiency in the production of ethyl esters (EE) under mild reaction conditions. However, its stability under different conditions has not been studied. Thus, it was determined that this CL produces EE in a stable way under different temperatures and in the presence of water and free fatty acids, by contrast, the addition of glycerol generated decreases of up to $70 \%$ in the synthesis of ethyl esters. Applying the Arrhenius model for a pseudo-first order reaction, an Ea of $21.4 \pm 1.4 \mathrm{~kJ} / \mathrm{mol}$ was determined, meaning a decrease of $5 \%$ compared to its main component (TLL-LW). Additionally, the use of a different oil (soybean) generated a $20 \%$ increase in EE production. Finally, a docking study and the kinetic simulation of the system were performed using a previously developed model, but a clear correlation with the experimental results was not achieved. Apart from this, due to its robustness, the CL studied has a high potential for application in synthesis of ethyl esters.
\end{abstract}

Keywords: Biocatalysts; Combi-lipases; Palm Oil; Docking; Ethyl Esters; Enzymes

\section{Efeitos de alguns parâmetros de reação na produção de ésteres etílicos usando uma lipase combinada de TLL e CALB no Lewatit VP OC 1600}

\begin{abstract}
Resumo
A otimização de misturas de lipases imobilizadas (combi-lipases) tem sido aplicada como estratégia para aumentar a velocidade e o rendimento na produção de ésteres alquílicos de ácidos graxos ou em óleos vegetais hidrolisados em comparação à biocatálise convencional (catalisadores monoenzimáticos). Anteriormente, a Combi-lipase (CL) da Thermomyces lanuginosus (TLL) e Candida antarctica isoforma B (CALB) nas proporções 75:25 no suporte Lewatit VPOC 1600 (LW) foram identificadas como as mais ativas com oleína de palma, de de acordo com a quantidade de proteína imobilizada e sua eficiência na produção de ésteres etílicos (EE) sob condições de reação moderada. No entanto, sua estabilidade em diferentes condições não foi estudada. Assim, determinou-se que este CL produz EE de forma estável sob diferentes temperaturas e na presença de água e ácidos graxos livres, ao contrário, a adição de glicerol gerado diminui em até $70 \%$ na síntese de ésteres etílicos. Aplicando o modelo de Arrhenius para uma reação de pseudo-primeira ordem, foi determinado um Ea de 21,4 $\pm 1,4 \mathrm{~kJ} / \mathrm{mol}$, significando uma diminuição de 5\% em comparação com seu componente principal (TLL-LW). Além disso, o uso de um óleo diferente (soja) gerou um aumento de $20 \%$ na produção dos EE. Finalmente, um estudo de ancoragem e a simulação cinética do sistema foram realizados usando um modelo previamente desenvolvido, mas uma correlação clara com os resultados experimentais não foi alcançada. Além disso, devido à sua robustez, o $\mathrm{CL}$ estudado tem um alto potencial de aplicação na síntese de ésteres etílicos.
\end{abstract}

Palavras-chave: Biocatalisadores; Combilipases; Óleo de Palma; Docking; Ésteres Etílicos; Enzimas. 


\section{Introducción}

Las lipasas han sido evaluadas en la producción de biodiésel mediante la transesterificación de aceites vegetales, la mayoría de ellas usando metanol debido a su bajo costo y fácil obtención. Sin embargo, la producción usando etanol es una alternativa más verde ya que puede ser obtenido de fuentes vegetales como el maíz y la caña de azúcar o con el uso de materias primas lignocelulósicas mediante un proceso de segunda generación $[1,2]$. Las lipasas son enzimas muy utilizadas en la biocatálisis debido a su capacidad de reconocer una amplia gama de sustratos [3], pero como sucede con otras enzimas, su costo sigue siendo un impedimento para su uso. Eso ha llevado a buscar diferentes estrategias para solventar esta barrera, entre ellas la inmovilización de las mismas en soportes macroporosos, de celulosa o mediante encapsulamiento, entre otras $[4,5]$, lo que facilita su separación del medio reacción.

En la producción de ésteres etílicos de ácidos grasos (EE), constituyentes principales del biodiesel etílico, la inmovilización de una lipasa en un soporte poroso genera ventajas frente a la catálisis convencional tales como: la posibilidad de reúso del biocatalizador, la disminución del gasto energético en la producción dada las bajas temperatura que oscilan entre 293 y $310 \mathrm{~K}$ comparadas con la convencional que se hace desde $323 \mathrm{~K}$ [4], menor exceso molar de etanol, así como un uso mínimo de solventes, contribuyendo a un proceso más amigable con el medio ambiente [5].

La optimización de mezclas de lipasas inmovilizadas (Combi-lipasas) ha sido aplicada como estrategia para incrementar la velocidad y el rendimiento en la producción de ésteres alquílicos de ácidos grasos o en aceites vegetales hidrolizados con respecto a la biocatálisis convencional basada en lipasas individuales [6,7]. En la mayoría de los casos, estos incrementos han sido atribuidos a la complementariedad catalítica de las lipasas involucradas, como puede ser la que se da entre una lipasa no específica y otra con especificidad sn-1 y sn-3 [6].

La implementación de la transesterificación basada en biocatalizadores lipásicos está limitada por la inactivación enzimática: puede darse por factores como el efecto inhibitorio del alcohol, susceptibilidad a la desnaturalización térmica, química y a cambios del medio de reacción, entre otros $[8,9]$. Dentro de los sistemas heterogéneos más usados, se ha reportado que Lipozyme ${ }^{\circledR} T L$ IM presenta una producción mayor al $80 \%$ de ésteres metílicos a una temperatura de $313 \mathrm{~K}$ [10]; sin embargo, cuando se emplea etanol como aceptor de acilo presenta una producción del $60 \%$ de EE requiriendo un exceso molar de etanol: aceite de 7:1 [11]. Por otro lado, estudios para CL basadas en Novozyme ${ }^{\circledR} 435$, Lipozyme ${ }^{\circledR} T L$ IM y mezclas de ellas, se evaluaron en presencia de ácidos grasos libres, presentando estabilidad e incluso disminución de la acidez del medio después de $4 \mathrm{~h}$ de reacción con rendimiento de hasta $50 \%$ de EE a $323 \mathrm{~K}$ [12]. Si bien, estos sistemas mostraron buenos resultados, al basarse en biocatalizadores heterogéneos comerciales no queda claramente definido el contenido de proteína o de actividad inmovilizada en cada uno de ellos haciendo difícil su comparación con sistemas lipásicos relacionados. Recientemente, en nuestro grupo fueron estudiados diferentes sistemas de Combi-lipasa (CL) basados entre otras, en la lipasa de Thermomyces lanuginose (TLL) y la lipasa isoforma B de Candida antarctica inmovilizadas en el soporte hidrofóbico Lewatit $\AA$ VP OC 1600 (LW) de modo sistemático: La CL más destacada fue aquella basada en TLLLW (75\%) y CALB-LW (25\%); esta fue obtenida minimizando el gasto de enzima (hasta del $80 \%$ para TLL) y mostrando propiedades interesantes como ser activa en la producción de EE de oleína de palma con rendimientos de EE hasta del $82 \%$ bajo condiciones muy suaves de reacción (310 K, relación etanol: oleína de palma 3,1:1) [13].

Las Combi-lipasas (como muchos otros catalizadores) se optimizan para ciertas condiciones muy específicas de reacción, pero no queda claro si son lo suficientes robustas cuando deben ajustarse a cambios, algunas veces inevitables, como los dados cuando varía la composición exacta del aceite natural usado de un lote a otro como por ejemplo en el contenido de agua y de ácidos grasos libres por mencionar algunos parámetros claves en la síntesis de biodiésel [14]. La finalidad de esta investigación fue observar no solo tales efectos en las propiedades de la $C L$ basa en TLL-LW (75\%) y CALB-LW (25\%) [13] sino de otros, como la variación de la temperatura, de la relación molar de etanol:aceite, de la adición de ésteres etílicos, glicerol e inclusive del cambio de aceite; estos efectos se evaluaron también sobre sus constituyentes individuales (lo que puede explicar las propiedades superiores observadas en ocasiones en los sistemas de Combi-lipasas) y 
frente a un catalizador lipásico de amplia relevancia comercial como lo es Novozyme® 435 [15].

\section{Metodología}

\section{Materiales}

Las lipasas de Candida antarctica isoforma B (CALB), Thermomyces lanuginosus (TLL), $p$-nitrofenilbutirato ( $p$-NFB), etanol y las sales utilizadas para la preparación de soluciones amortiguadoras fueron adquiridas de Sigma Chem. Co. (St. Louis, MO, USA). La oleína de palma y el aceite de soya fueron comprados en un local comercial de la ciudad y caracterizados por GC-MS (tabla 1) [13,16]; el catalizador Novozyme ${ }^{\circledR}$
435 fue un obsequio de Novozymes (Bagsværd, Dinamarca); el soporte comercial de Lewatit $^{\circledR}$ VP OC 1600 fue generosamente donado por Lanxess ${ }^{\circledR}$ (Colonia, Alemania).

\section{Obtención de los derivados lipásicos}

El soporte se acondicionó mediante lavados con etanol $(50 \% \mathrm{v} / \mathrm{v})$ y la solución de inmovilización sin enzima compuesta por glicerol (20 \%v) EDTA $(1 \mathrm{mM})$, azida de sodio $(0,05 \% \mathrm{v})$ y buffer fosfato de sodio $(25 \mathrm{mM})$ ajustando el $\mathrm{pH}$ a 7,0 . Posteriormente, se adicionó la solución de inmovilización con enzima y se realizó seguimiento mediante la hidrólisis de $p$-NFB durante 24 h [13].

Tabla 1. Composición porcentual de los ácidos grasos presentes en la oleína de palma y aceite de soya.

\begin{tabular}{cccc}
\hline Ácido graso & Estructura & $\begin{array}{c}\text { Oleína Palma } \\
\text { Composición, \% }\end{array}$ & $\begin{array}{c}\text { Aceite de soya } \\
\text { Composición, \% }\end{array}$ \\
\hline Palmítico & $16: 0$ & 67,93 & 23,04 \\
Linoleico & $18: 2$ & 16,04 & 44,23 \\
Oleico & $18: 1$ & 14,28 & 25,45 \\
Esteárico & $18: 0$ & 1,76 & 7,28 \\
\multicolumn{2}{c}{ Aceite PM, g/mol } & 831,54 & 919,22 \\
\hline
\end{tabular}

\section{Actividad enzimática y determinación de proteína}

La actividad hidrolítica se cuantificó mediante la unidad internacional (UI), que se define como la cantidad de enzima requerida para hidrolizar 1 $\mu \mathrm{mol}$ de $p$-NFB. min $^{-1}$; esta reacción se desarrolló a $\mathrm{pH} 7,0$ y $298 \mathrm{~K}$ con posterior detección en un espectrofotómetro Biomate 35 a una longitud de onda de $348 \mathrm{~nm}$. Para CALB se adicionó una mezcla de detergentes al 0,01 y $0,0016 \%$ v de Tritón ${ }^{\circledR}$ $\mathrm{X}-100$ (TX) y CTAB respectivamente, mientras que para TLL se requirió TX al 0,015\%v[17].

Para el cálculo de los porcentajes de inmovilización se tiene en cuenta la actividad residual del sobrenadante mediante la ecuación:

$$
100 \%\left(1-X_{S S} \cdot X_{C S}^{-1}\right)=\% \text { inmovilizado }
$$

Donde $X_{S I}$ y Xcs indican la actividad hidrolítica (o la cantidad proteína) después de cada muestreo en los sobrenadantes que estaban en contacto antes de las medidas sin (SS) y con soporte (CS) de inmovilización respectivamente.
La proteína se determinó acorde al kit Pierce $₫$ $\mathrm{BCA}$ a una temperatura de $310 \mathrm{~K}, 600 \mathrm{rpm}$ y una incubación de 30 min en un Thermomixer ${ }^{\circledR}$ y se cuantificó utilizando un espectrofotómetro Biomate $3 S$ a una longitud de onda de $562 \mathrm{~nm}$. La proteína inmovilizada se determinó por la diferencia entre el contenido de proteína del control y del sobrenadante de inmovilización después de $24 \mathrm{~h}$ [13].

\section{Obtención de combi-lipasas}

La combi-lipasa se obtuvo por el pesado y la mezcla de los constituyentes adecuadamente secados en proporciones (\%p) 75:25; cada componente individual se optimizó en cuanto a la carga de proteína, obteniendo para TLL y CALB un valor de $15,6 \pm 0,55$ y $69,12 \pm 2,74 \mathrm{mg}^{*} \mathrm{~g}^{-1}$ proteína inmovilizada respectivamente. Por último, se determinó la actividad hidrolítica inmovilizada la cual fue del $73,26 \pm 1,80 \%$ para TLL y 94,42 $\pm 4,58 \%$ para CALB y se procedió a evaluar su eficiencia en la producción de ésteres etílicos. 


\section{Producción de ésteres etílicos (EE)}

Las condiciones de referencia [13] para la obtención de EE fueron $310 \mathrm{~K}$, relación molar etanol:aceite 3,1:1, $1600 \mathrm{rpm}$ y proporción del biocatalizador con respecto al aceite de 5,75\%p. En otros experimentos se varió la temperatura entre 298 y $333 \mathrm{~K}$ sin modificar las demás condiciones anteriormente descritas. Para la evaluación del efecto de la presencia de agua, se adicionó en una mezcla con etanol absoluto manteniendo la relación molar etanol: aceite 3.1:1 y variando el porcentaje de agua en la mezcla en $0,4,4,0,7,0$, 10,0 y $13,0 \%$ v. Posteriormente, se varió la relación molar etanol:aceite en proporciones 1:1, 2:1, 3,1:1 y 6:1 conservando las condiciones iniciales de temperatura y cantidad del biocatalizador.

Al evaluar la adición de glicerol se mantuvieron constantes los parámetros iniciales y se realizó una mezcla del glicerol con el alcohol manteniendo la relación molar etanol:aceite y variando el glicerol en porcentajes de $12,5,25,36,5$ y $50 \%$; este porcentaje se basa en la producción teórica que se espera según la cantidad de aceite utilizado en la reacción de transesterificación. El biodiesel químico fue adicionado al 12,3, 19,2 y 33,6 \%p garantizando la proporción catalizador:aceite.

Por último, mediante el uso de atmósfera inerte, se preparó la mezcla de ácido palmítico y oleico en relación porcentual en peso de 52:48, acto seguido se mezcló con el aceite y se obtuvieron soluciones de $1,25,2,5,3,75$ y $5,0 \%$ que se adicionaron al vial de reacción, respetando la relación molar etanol:aceite inicial.

La determinación de ésteres etílicos de ácidos grasos se llevó a cabo mediante ATR-FTIR y la herramienta estadística Rstudio; los datos fueron adquiridos realizando tres experimentos independientes por cada muestra. Se realizó una prueba t-Student con un 95\% de confianza para evaluar la diferencia significativa entre los valores obtenidos en el desarrollo de la investigación.

\section{Métodos docking}

Se usó el software PyRx 3.3 [18] con las estructuras de las proteínas del Protein Data Bank PDB 5A71 para CALB y 1DTE para TLL que corresponden al confórmero abierto de cada lipasa. En los dos casos, la exhaustividad usada fue de 10. La GridBox se ubicó de modo tal que se cubrieran los lids y el bolsillo catalítico de cada lipasa.

\section{Resultados y Discusión}

Selección del mejor sistema combi-lipasa para el estudio comparativo de los efectos de diferentes parámetros de reacción

Se realizó una comparación entre el derivado coinmovilizado y la combilipasa de TLL y CALB en LW, y aunque la coCL (sistema de lipasas coinmovilzadas) fue más activa que la $\mathrm{CL}(89,5 \pm 3,4$ y $81,8 \pm 0,7$ respectivamente) [13] fue seleccionado el derivado CL de TLL y CALB $(75: 25)$ ya que permite realizar una comparación más exacta entre los diferentes efectos frente a sus derivados constituyentes; la $\mathrm{coCL}$ introduciría efectos adicionales por el hecho de haber una interacción entre dos enzimas en una misma superficie del catalizador.

\section{Efecto de la temperatura en la producción de EE usando la CL TLL:CALB}

Por lo anteriormente dicho, se procedió a evaluar el efecto de la temperatura en la síntesis de EE para la CL compuesta por $75 \%$-TLL y $25 \%$-CALB en LW, usando como referencia los derivados monolipásicos y Novozyme ${ }^{\circledR}$ 435. En cada uno de los casos se observa a las 24 h una disminución de la producción de EE cuando la reacción se lleva a cabo a $333 \mathrm{~K}$, inclusive para CALB se observa un comportamiento constante que no supera el $12 \%$ de $\mathrm{EE}$, mientras que a $323 \mathrm{~K}$ permite un aumento del $47 \%$ en la producción de biodiésel respecto a la máxima temperatura (figura 1). La combi-lipasa presenta una velocidad de conversión mayor entre las 4 y $6 \mathrm{~h}$ a medida que la temperatura aumenta, después se estabiliza sin un aumento considerable en la producción de EE a temperaturas entre los 310 y $333 \mathrm{~K}$; sin embargo, a $298 \mathrm{~K}$ si hay un comportamiento creciente con el tiempo, aunque inferior en las primeras horas, pero superando en un $6 \%$ al derivado estudiado a $333 \mathrm{~K}$ (figura 1 ).

La referencia comercial Novozyme ${ }^{\circledR} 435$ presentó gran afectación a $333 \mathrm{~K}$ disminuyendo un 50\% su rendimiento con respecto a la temperatura de referencia $(310 \mathrm{~K})$, adicionalmente, a $323 \mathrm{~K}$, presentó medidas muy variables con \%RSD (Porcentaje de Desviación estándar relativa) mayores al $20 \%$ a las $24 \mathrm{~h}$; los valores máximos de producción de EE se ven en la tabla 2 para cada derivado. 
rev. ion. 2020;33(2):83-97. Bucaramanga (Colombia).

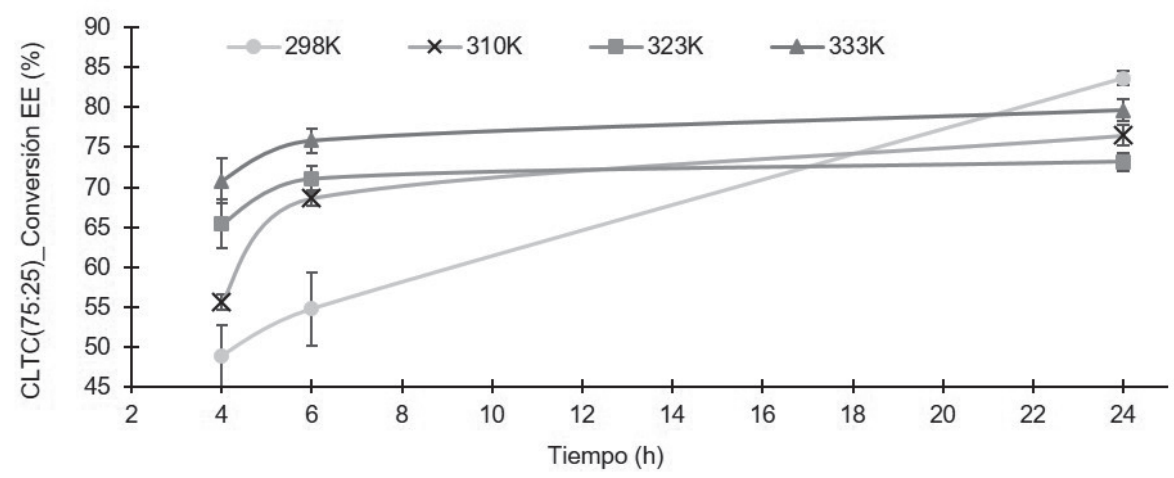

Figura 1. Conversión de EE de la combi-lipasa de TLL y CALB $(C L)$ a diferentes temperaturas.

Tabla 2. Valores de producción de EE a partir de oleína de palma a las 24 h de reacción.

\begin{tabular}{ccc}
\hline Derivado & $\% \mathrm{EE}_{\max }(\mathrm{T}, \mathrm{K})$ & $\% \mathrm{EE}_{\min }(\mathrm{T}, \mathrm{K})$ \\
\hline $\mathrm{CALB}$ & $28,79 \pm 1,56(323)$ & $13,48 \pm 0,67(333)$ \\
$\mathrm{TLL}$ & $87,08 \pm 0,89(298)$ & $70,80 \pm 4,66(333)$ \\
$\mathrm{CL}(75: 25)$ & $83,66 \pm 0,87(298)$ & $73,16 \pm 1,13(323)$ \\
Novozyme $^{\circledR}$ & $33,08 \pm 1,86(310)$ & $16,27 \pm 1,49(333)$ \\
\hline
\end{tabular}

Durante el transcurso de la reacción se puede observar en todos los casos que la velocidad de conversión aumenta con la temperatura durante las primeras $6 \mathrm{~h}$ de reacción (figura 2), esto puede ser debido a que la solubilidad del aceite en el etanol aumenta disminuyendo fenómenos de transferencia de masa que pueden ralentizar la reacción [9]; esta homogenización es favorecida por los di- y mono-glicéridos que se van produciendo en la medida que avanza la reacción, facilitando la difusión del alcohol para que se lleve a cabo la reacción en la superficie activa del biocatalizador, sin embargo, al aumentar el tiempo de exposición del biocatalizador a una temperatura superior a los $310 \mathrm{~K}$ se podría estar presentando la inactivación de la enzima causando disminución en la producción de EE [10]. En la figura 2 está representado el comportamiento esperado de manifestarse en la $\mathrm{CL}$ un efecto meramente aditivo respecto a sus constituyentes mono-enzimáticos (línea punteada); para cuantificar estas desviaciones a la aditividad se emplea el factor de complementariedad (ecuación 2), FC [13]:

Un FC $=1$, indica que el efecto que se presenta en la $C L$ es meramente aditivo, mientras que un FC > 1 permite deducir que existe un fenómeno de sinergia entre los biocatalizadores que componen la $\mathrm{CL}$, lo contrario ocurre si $\mathrm{FC}<1$ ya que indica que la eficiencia de la $C L$ disminuye por efectos anti-sinérgicos al combinarse [13].

$$
F C_{C_{1}, C_{2}}=\frac{\% E E_{C o m b i}\left(C_{1}, C_{2}\right)}{\% E E_{\text {calc }}\left(C_{1} / 100 * \% \mathrm{EE}_{1}+C_{2} / 100 * \% \mathrm{EE}_{2}\right)}
$$

Donde $\% \mathrm{EE}_{\mathrm{Combi}}$ es la producción medida a las 24 $\mathrm{h}$ de la combi-lipasa binaria con un porcentaje de composición $C_{1}$ y $C_{2}$; el $\% \mathrm{EE}_{\text {calc }}$ resulta de la suma ponderada del \%EE a las $24 \mathrm{~h}$ para cada componente individual bajo las mismas condiciones de reacción.

En el caso del efecto de la $\mathrm{T}$ vemos que la $\mathrm{CL}$ presenta valores crecientes de FC desde 1,2 hasta 
1,7 , con un $\%$ RSD inferior al $5 \%$, a medida que aumenta la temperatura. Lo que sugiere efectos sinérgicos donde se complementa la estabilidad de TLL ante la inactivación de CALB durante el aumento de temperatura.

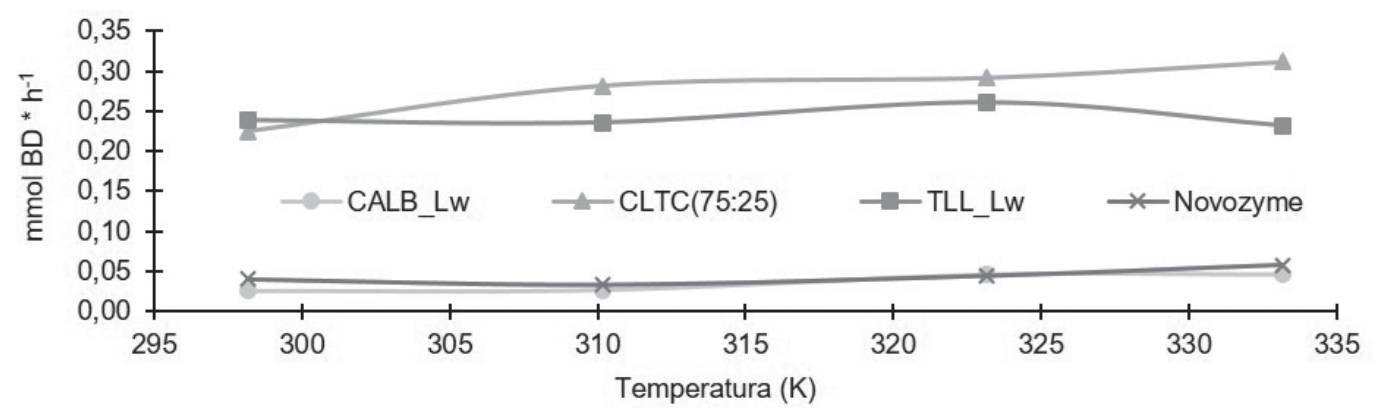

Figura 2. Velocidad de conversión a las 6 h de reacción a diferentes temperaturas de los biocatalizadores usados.

De otro lado, en la reacción de la conversión de triglicéridos (TAG) a ésteres de ácidos grasos suceden tres etapas reversibles implicando diglicéridos (DAG) y mono-glicéridos (MAG) como intermediarios: se ha observado que Novozyme ${ }^{\circledR}$ 435 transesterifica sin una acumulación significativa de TAG o DAG, indicando que la conversión de TAG en DAG para este biocatalizador es el paso limitante de la reacción. Así, con el objetivo de determinar la energía aparente de activación de la reacción, se analiza la velocidad en el consumo de TAG durante la producción enzimática de EE a diferentes temperaturas [1].

Para analizar los datos obtenidos se aplicó el modelo de Arrhenius (ecuación 3 y 4), que permite relacionar la dependencia de las constantes de velocidad de la reacción con la temperatura y estimar los cambios en la energía de activación del sistema [1].

$$
k=A e^{\wedge}(-E a / R T)
$$

$$
\operatorname{In}(k)=\operatorname{In}(A)-(E a / R)(1 / T)
$$

Donde A es un factor pre-exponencial, $E a$ corresponde a la energía de activación y $\mathrm{k}$ es la constante de velocidad de primer orden. Para los biocatalizadores estudiados se obtuvieron correlaciones con un intervalo de valores de $R^{2}$ entre 0,82 - 0,96 y energías de activación (figura 3) de 22,51,5, 21,51,5, 17,81,2 y $21,41,4 \mathrm{~kJ} / \mathrm{mol}$ para TLL, CALB, Novozyme ${ }^{\circledR} 435$ y la CL respectivamente; estos valores obtenidos presentan diferencias inferiores al $15 \%$ respecto a estudios en la transesterificación de aceite de sardina para TLL [18] y aceite de algodón para CALB y Novozyme ${ }^{\circledR} 435$ [22], adicionalmente, se observó una disminución significativa ( $t_{\text {calculado }}$ $=3,78>t_{\text {critico }}=2,78$ ) del $5 \%$ en la $E a$ de la $C L$ con respecto a su componente principal TLL, que puede ser atribuido a la sinergia que se presenta al combinar dos derivados de diferente enzima.

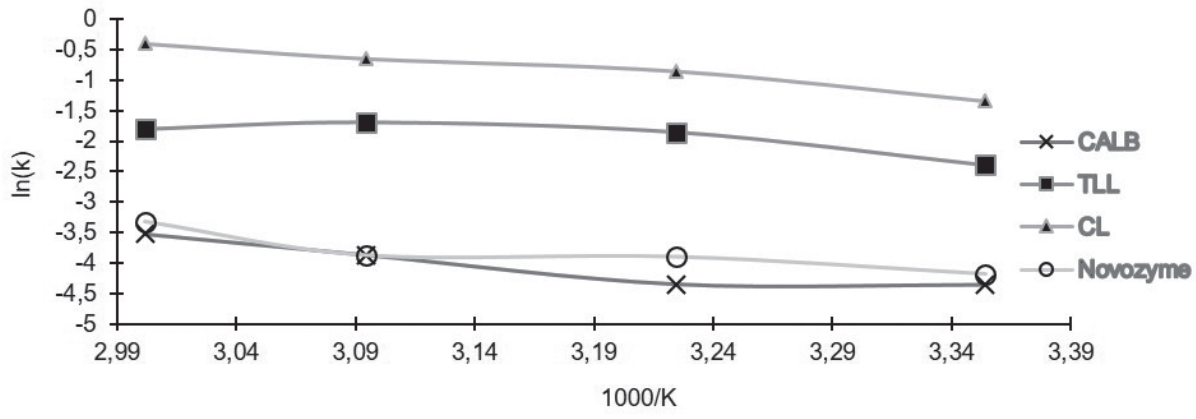

Figura 3. Análisis de la energía de activación mediante linealización de la ecuación de Arrhenius. 
Adicionalmente, puede indicar mejores rendimientos en tiempos más cortos de reacción para la CL. El principal factor en los porcentajes de error encontrados se puede atribuir a que el modelo de Arrhenius no tiene en cuenta la inactivación enzimática [1] o la desorción de la misma de la partícula de soporte, como se evidenció en los estudios previos de reúso para estos sistemas [13].

\section{Efecto de la relación molar etanol:aceite en la producción de EE}

La relación molar del alcohol respecto al aceite es uno de los factores que afecta hasta en un $60 \%$ la eficiencia de conversión de EE, a su vez, el costo de producción del biodiesel se ve afectado debido a que la relación molar usada en la industria llega incluso a ser de 10:1, que constituye un riesgo alto para el personal de la planta dado su volatilidad e inflamabilidad [23]. En la CL TLL-LW+CALB-LW (75:25) este efecto fue evaluado y se comparó con la referencia comercial Novozyme ${ }^{\circledR} 435$ y sus derivados mono-lipásicos constituyentes: se observó que el combi-catalizador no parece afectarse de manera considerable por el etanol (figura 4), incluso mostrando valores de FC desde 1,05 a 1,17 , con $\% R S D$ inferiores al $6 \%$, indicando sinergia. Sin embargo, el uso del alcohol en varios ciclos de reacción sí puede afectar la eficiencia del catalizador según resultados previos en este sistema [13].

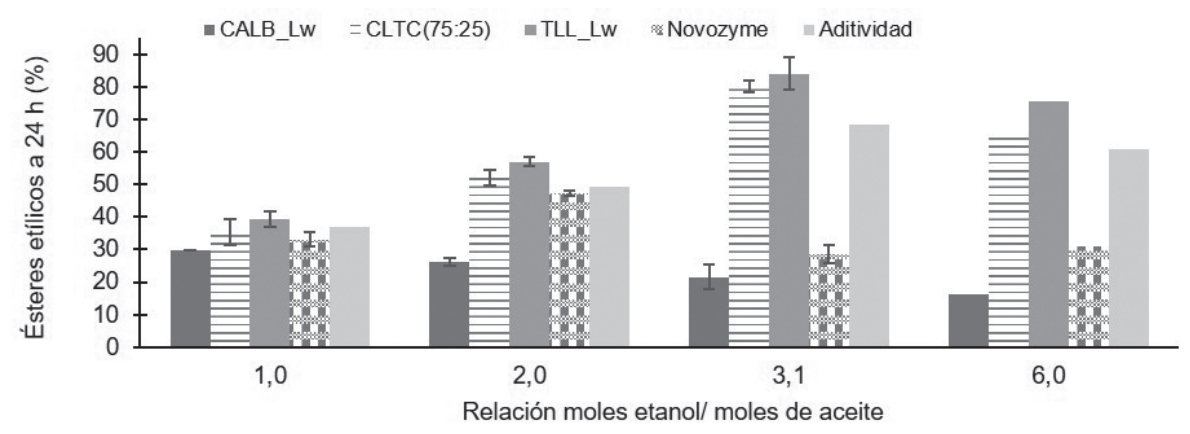

Figura 4. Efecto de la relación molar etanol:aceite en la producción de EE a las 24 h de reacción.

Como se deduce de la estequiometría de la reacción, el etanol es el reactivo limitante en relaciones molares menores a $3: 1$, esto se pudo observar experimentalmente (tabla 3) pues al aumentar gradualmente la cantidad se generó un óptimo al tener una relación molar 3,1:1 etanol:aceite. No obstante, por encima de ese valor se genera una disminución en el rendimiento de EE de hasta el 20 $\%$ en la producción de ésteres etílicos. Esto porque el etanol afecta la estructura proteica inactivando la enzima, algo observado en sistemas similares bajo relaciones molares 6:1 [23].

Tabla 3. Valores máximos y mínimos de \%EE para los derivados evaluados.

\begin{tabular}{|c|c|c|}
\hline Derivado & \%EEmax ( $\left.{ }^{a}\right)$ & $\%$ EEmin $\left({ }^{a}\right)$ \\
\hline CALB & $29,86 \pm 0,49(1: 1)$ & $16,26 \pm 3,85(6: 1)$ \\
\hline TLL & $84,13 \pm 1,47(3: 1)$ & $39,31 \pm 0,65(1: 1)$ \\
\hline CLTC(75:25) & $80,12 \pm 2,32(3: 1)$ & $35,36 \pm 0,86(1: 1)$ \\
\hline Novozyme $^{\circledR}$ & $47,23 \pm 2,18(2: 1)$ & $30,86 \pm 2,22^{b}$ \\
\hline
\end{tabular}

En contraste con los catalizadores inorgánicos, los biocatalizadores pueden funcionar adecuadamente sin excesos molares de etanol; incluso en relaciones molares $1: 1$ se produce el máximo valor teórico, que es lo que se aplica en la estrategia de síntesis de adición por partes, pudiendo llegar a rendimientos inclusive del $99 \%$ de EE junto con estrategias para retirar productos (EE y glicerol) y la adición de reactivos consumidos, algo que se fundamenta en el principio de Le Châtelier y en la protección de la enzima ante la inactivación causada por el aceptor de acilo $[24,25]$. 
Efecto del porcentaje de agua presente en la reacción

Las enzimas requieren cierto nivel de agua en su estructura para mantener su conformación activa, ya que el agua confiere la flexibilidad requerida para los cambios conformacionales que se dan durante el proceso catalítico. En contraste, esta puede modificar las propiedades de solubilidad de los reactivos y productos llegando incluso a inactivar la enzima a altas concentraciones, más aún, el exceso de agua entraría a competir con el etanol como aceptor de acilo llevándose a cabo la reacción de hidrólisis compitiendo con la de transesterificación [26,27].

Como se observa en la figura 5, los biocatalizadores de TLL y la CL presentaron una gran estabilidad frente a la presencia de agua en el medio de reacción; esto supone poder ampliar el uso de estos sistemas en la transesterificación de aceites poco refinados o etanol con un contenido de agua relativamente alto; esto contribuye en disminuir la inversión asociada en materias primas refinadas que constituyen hasta en un $30 \%$ del costo del proceso sugiriendo la posibilidad de aplicar la CL para aprovechar aceites usados [28].

Por el contrario, CALB en LW y Novozyme ${ }^{\circledR} 435$ presentaron una disminución de hasta en un $20 \%$ EE al incrementar la cantidad de agua; esto ha sido asociado a la formación de complejos dentro del catalizador ya que las moléculas de agua generan una barrera hidrofílica alrededor de la enzima impidiendo la difusión del substrato hidrofóbico (triglicérido) de la fase oleosa hacia el sitio activo de la enzima [15,29]; adicionalmente, la actividad hidrofílica $\left(a_{w}\right)$ juega un papel importante en los resultados obtenidos, este valor fluctúa entre 0 para un sistema completamente libre de humedad y 1 para el sistema saturado de agua [30]. CALB presenta su actividad enzimática óptima en medios orgánicos a valores de $a_{w}=0$, mientras que TLL, acepta rangos dependiendo del medio de reacción, desde 0,77 a 0,97 ; justificando el mayor rendimiento de TLL-LW y de la CL frente a Novozyme ${ }^{\circ} 435$ y CALB.

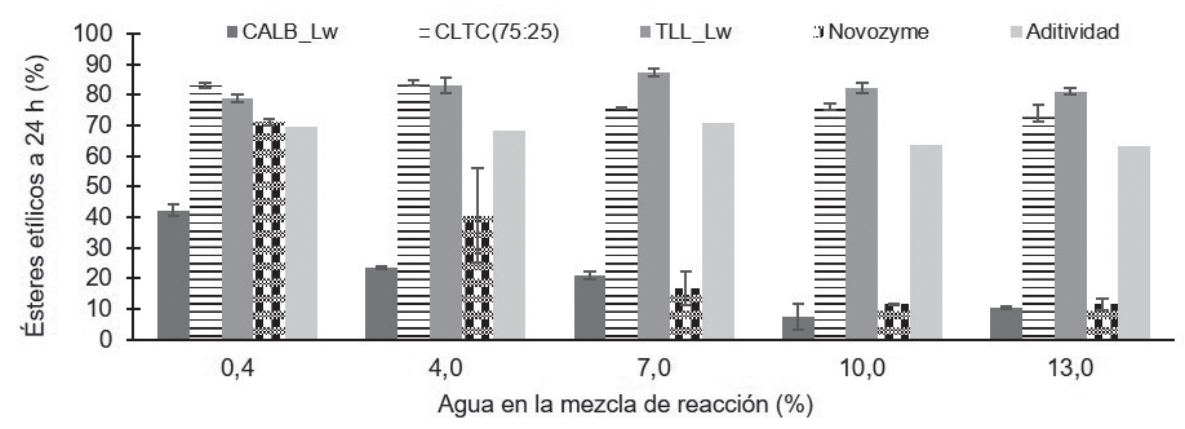

Figura 5. Efecto de la presencia de agua en la producción de EE a las 24 h de reacción.

Efecto de la presencia de glicerol en la transesterificación de oleína de palma

El glicerol es un subproducto de la reacción de transesterificación y eventualmente puede inhibir el avance de la misma por consideraciones de equilibrio. Este compuesto implicó una disminución gradual en el rendimiento del 80 al $21 \%$ EE al añadirlo desde el comienzo de la reacción (figura 6); estos cambios son congruentes con lo observado en sistemas similares pues éste podría formar una capa hidrofílica que recubre la superficie del biocatalizador afectando la difusión de los reactantes (en especial los TGs por su hidrofobicidad) hacia el sitio catalítico de la enzima, lo que se traduce en una menor producción de EE [31]. Al igual que con los efectos anteriores, se presentó un comportamiento muy similar de la CL comparado con su constituyente de TLL-LW, algo que se relaciona con el hecho de que este es el componente principal de la $C L$ [15]. EL factor de complementariedad para la CL se mantiene constante con un valor promedio

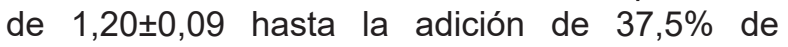
glicerol donde se encontró una disminución en la producción de ésteres etílicos del 30\% y valores del $F C<1$, mostrando la influencia de TLL en la $C L$ ya que el derivado individual también presenta disminuciones hasta del $45 \%$ después de la adición de grandes cantidades de glicerol.

Que el glicerol tuviera un relativo alto impacto en el rendimiento indicaría que el producido endógenamente por la reacción podría ser en parte el responsable de que no se logren rendimientos superiores a los obtenidos en este 
trabajo, sin embargo, superaron el $80 \%$ para la $C L$. Así, se podría aplicar estrategias de modificación del soporte o del medio de reacción mediante aditivos que inhiban la formación de tales capas de glicerol, tal como se ha hecho previamente con el uso de tert-butanol; la desventaja de añadir este solvente orgánico es incluir un volumen muerto en la mezcla de reacción, haciendo el proceso menos eficiente y verde [32].

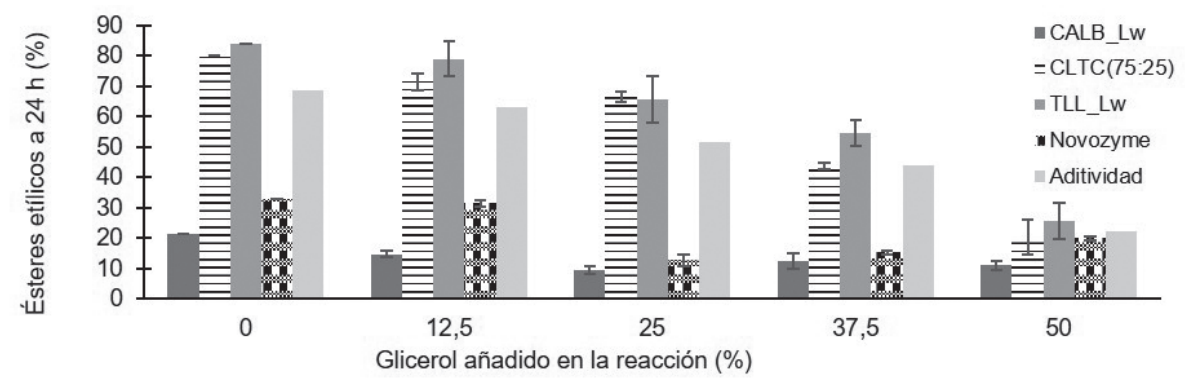

Figura 6. Efecto de la presencia de glicerol en la producción de EE a las 24 h de reacción.

Efecto de la adición de biodiésel en la reacción de transesterificación

En los procesos de biocatálisis para la producción de ésteres de ácidos grasos, se ha propuesto la adición de biodiésel como co-solvente para favorecer la solubilidad del alcohol en el aceite mejorando los procesos de difusión en el medio de reacción [15], por contraste, en este estudio se observó una disminución en la producción de EE (figura 7), de modo que al igual que con el glicerol, sería el principio de Le Châtelier el que explicaría la disminución en el rendimiento.

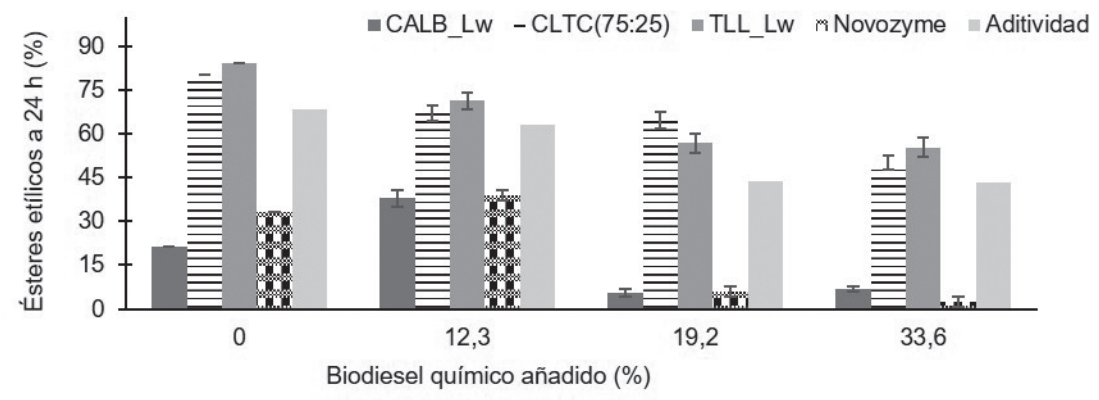

Figura 7. Efecto de la presencia de biodiesel químico en la producción de EE a las 24 h de reacción.

Hay que notar que el efecto del biodiesel es menos marcado que el del glicerol (reducción hasta 60 $\% \mathrm{EE})$, pues ya que, a diferencia del último, para este no se ha reportado que forme capas que impidan la difusión de los reaccionantes alrededor de la partícula del biocatalizador, lo que se puede atribuir a su naturaleza más hidrófoba. Novozyme ${ }^{\circledR}$ 435 presentó una disminución del $73 \%$ en su eficiencia en la producción de ésteres etílicos y se obtuvieron FC de 1,06 a 1,33, con \%RSD inferiores al $5 \%$, para la CL estudiada mostrando diferencias significativas en la tasa de producción de EE entre la $\mathrm{CL}$ y sus derivados individuales.

Efecto de la presencia de ácidos grasos libres en la producción de EE

El contenido de ácidos grasos libres (AGL) en el aceite juega un papel muy importante en la catálisis industrial, ya que son causantes de saponificación (cuando se usa catálisis básica). Esto supone la necesidad de aceites vegetales refinados cuyo contenido de $A G L$ sea inferior al $5 \%$ de AGL $[33,34]$, implicando un aumento en el costo de producción del biocombustible y un diseño de planta más complejo. En contraposición, para lipasas como CALB, los AGL puede constituir una materia prima en la síntesis de EE mediante esterificación con el alcohol, siendo una ventaja frente al resto de sistemas catalíticos [13]. Por lo anterior, se evalúo el comportamiento en la producción de ésteres etílicos para la mejor CL y sus componentes individuales de la adición de AGL (figura 8). 


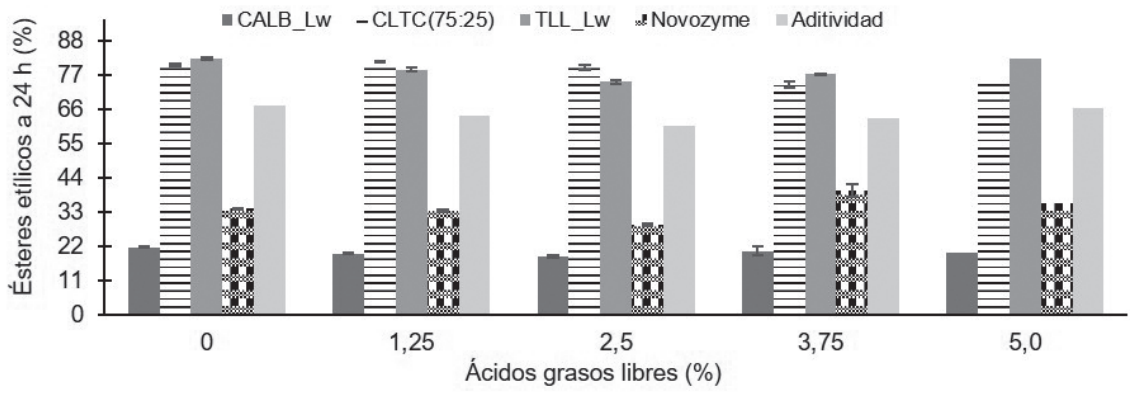

Figura 8. Efecto de la presencia de AGL en la producción de EE a las 24 h de reacción.

Los resultados obtenidos muestran que la CL (con un FC promedio de 1,10 \pm 0,01) y demás biocatalizadores son robustos ante la presencia de AGL, esto, junto con el hecho de la poca afectación ante la presencia de agua en el rendimiento, sugiere un potencial de aplicación de esta CL para la síntesis de EE provenientes de aceites usados o de baja calidad que suelen contener niveles elevados de estos compuestos [32,35], como se ha observado para Novozyme $\mathbb{R} 435$ en la producción de biodiesel a partir de aceites reusados con alto contenido de $A G L$ (6,4 \%p), donde se ha demostrado que puede realizar procesos de transesterificación y esterificación simultáneamente siendo hasta dos veces más rápida la transesterificación que la esterificación sin afectar su rendimiento [36].

Efecto del tipo de aceite en la producción de EE a partir de la CL de TLL y CALB en LW Los biocatalizadores basados en lipasas tienen la capacidad de actuar en diversos sustratos y la composición de estos puede afectar el rendimiento en la producción de ésteres alquílicos de ácidos grasos en especial cuando se trata de materias primas de origen natural; para observar este efecto se evalúo las CL de TLL y CALB en LW frente al aceite de soya, que es una de las materias primas más utilizadas en Irán, Argentina, Brasil e incluso China [37].

Al comparar los valores obtenidos entre el biodiésel a partir de oleína de palma y aceite de soya (figura 9) se observó un incremento hasta de un $20 \%$ en la producción de EE a partir del aceite de soya e incluso el factor de complementariedad aumento de 1,09 a 1,18 para la CL de TLL (75\%) y CALB (25\%); estudios con derivados de RML en diferentes soportes también evidenciaron incrementos del $15 \%$ en el rendimiento de la reacción con aceite de soya [16].

Estos incrementos serían consecuencia de la mayor polaridad del aceite de soya frente a la oleína de palma al ser más rico en triglicéridos insaturados, esto le permite disolver en mayor medida al etanol incrementando la velocidad de reacción. En paralelo, esto disminuiría la formación de microgotas inactivantes de etanol y la desorción de la enzima del soporte, pues este aceite competiría menos por la superficie del mismo que el de palma $[8,24]$. De hecho, en nuestro grupo se ha visto que CL similares de TLL y CALB (proporciones de 25:75 y 50:50 en LW) son hasta un $33 \%$ más estable en reúso con aceite de soya que en oleína de palma (datos no publicados).

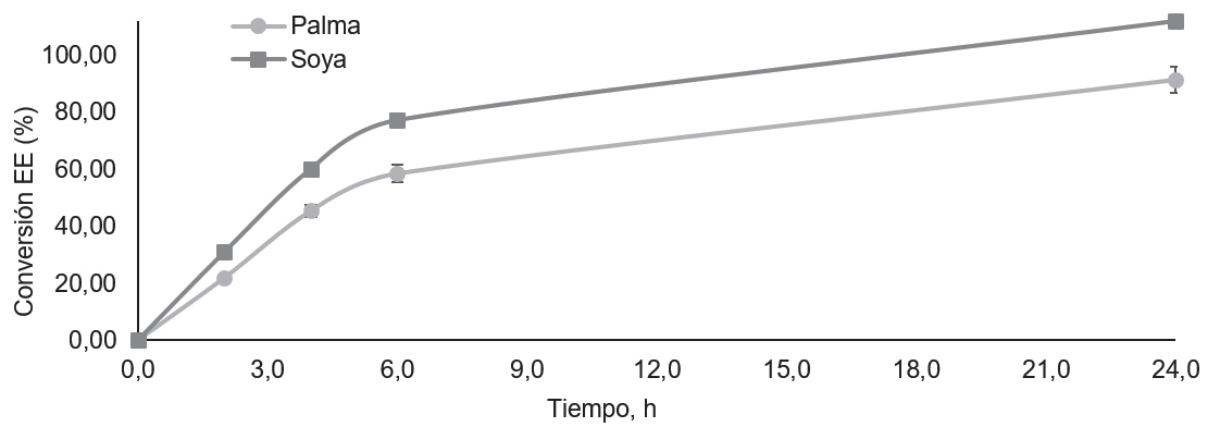

Figura 9. Efecto del tipo de aceite en la conversión de EE de la CL evaluada. 
Estudios de docking y simulación de un modelo cinético

Con el objetivo de explicar la tendencia observada por los biocatalizadores obtenidos respecto a su eficiencia en transesterificación de ésteres etílicos se utilizó el software PyRx 3.3 basado en autodockvina [18], para estimar la energía de afinidad de las enzimas por diferentes sustratos y de componentes como el glicerol, agua, ácidos grasos libres y etanol. TLL tiende a ser levemente más afín por los nucleófilos (agua, glicerol o etanol), mientras CALB es más afín por ésteres etílicos o AGL que por los triglicéridos (tabla 4).

Tabla 4. Afinidad de enlace con diferentes ligandos para TLL y CALB.

\begin{tabular}{|c|c|c|c|c|c|}
\hline Enzima & ligando & $\begin{array}{c}\text { Afinidad de } \\
\text { enlace }(\mathrm{kJ} / \mathrm{mol})\end{array}$ & Enzima & ligando & $\begin{array}{c}\text { Afinidad de } \\
\text { enlace }(\mathrm{kJ} / \mathrm{mol})\end{array}$ \\
\hline \multirow{10}{*}{$\sqsupset$} & Ácido oleico & $-25,121$ & \multirow{10}{*}{$\stackrel{n}{\frac{n}{d}}$} & Ácido oleico & $-24,702$ \\
\hline & Ácido palmítico & $-23,446$ & & Ácido palmítico & $-23,027$ \\
\hline & Palmitato de etilo & $-22,609$ & & Palmitato de etilo & $-22,609$ \\
\hline & Oleato de etilo & $-23,027$ & & Oleato de etilo & $-23,027$ \\
\hline & $\mathrm{POP}^{\mathrm{a}}$ & $-23,027$ & & POP & $-21,771$ \\
\hline & $\mathrm{POO}^{\mathrm{b}}$ & $-23,446$ & & $\mathrm{POO}$ & $-19,678$ \\
\hline & $\mathrm{PLO}^{\circ}$ & $-22,190$ & & PLO & $-21,71$ \\
\hline & Glicerol & $-16,747$ & & Glicerol & $-15,491$ \\
\hline & Etanol & $-11,304$ & & Etanol & $-10,467$ \\
\hline & Agua & $-7,118$ & & Agua & $-7,955$ \\
\hline
\end{tabular}

a1,3-palmitoil-2-oleilglicerol; b1-palmitoil-2,3-dioleilglicerol; ‘ 1-palmitoil-2-linoleil-3-oleilglicerol

Es importante mencionar, que los valores de la tabla 4 corresponden a poses del ligando donde el grupo carboxilo esta direccionado hacia el residuo de serina activo (en rojo), en coherencia con el mecanismo catalítico de estas serina-hidrolasas. Aunque TLL presenta un bolsillo catalítico más grande que CALB, la energía con que se estabiliza

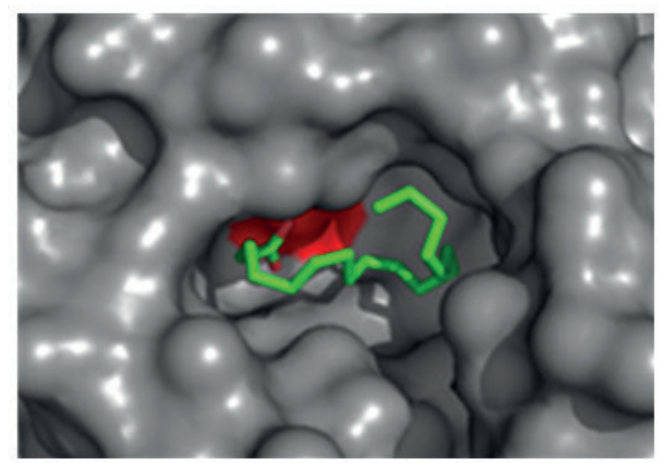

a) el AGL en los dos casos es similar $(-25 \mathrm{~kJ} / \mathrm{mol})$; este patrón también se observa con el resto de ligandos ensayados. La diferencia más notoria es con el triglicérido 1-palmitoil-2,3-dioleilglicerol (el $36 \%$ del total en el aceite[39]) llegando a ser de $-3,8 \mathrm{~kJ} / \mathrm{mol}$ a favor de TLL.

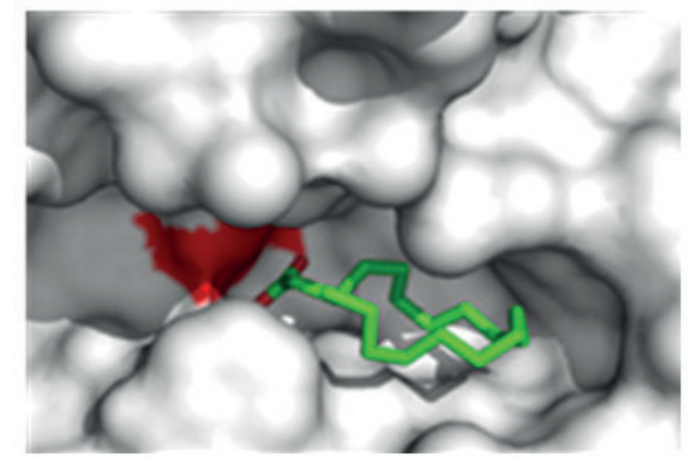

b)

Imagen 1. Resultados de docking para a) CALB (PDB 5A71)-ácido oleico y b) TLL (PDB 1DTE)-ácido oleico en su complejo más estable. Se empleó el programa Pymol 0.99rc6 para la visualización de los resultados. En color rojo se muestra la superficie del residuo Ser que hace parte de la triada catalítica de cada enzima y en verde el ácido oleico (sustrato) 
Aunque la herramienta de docking no puede simular aspectos del sistema real de reacción estudiado, como la superficie del soporte de inmovilización, la mezcla de reacción cambiante durante el proceso y su heterogeneidad, resultan una aproximación adecuada al explicar la mayor actividad de TLL en síntesis de EE frente a CALB de acuerdo con lo hallado experimentalmente.

De otro lado el modelado cinético fue realizado mediante el software Kinetoscope 1.1.8 empleando los mecanismos y parámetros de reacción estudiadas previamente [13]. En general, los resultados modelados obtenidos (tabla 5) no se ajustan al comportamiento experimental, por ejemplo, el modelo predice inhibición de TLL-LW por los AGL (hasta en un 52\%) pero esto no se evidenció en este estudio. La razón de esto se puede atribuir a algunos limitaciones del modelo como no incluir la formación de capas inhibitorias de difusión de agua o glicerol y otras limitaciones mencionadas previamente como la inactivación o desorción de la enzima desde la partícula del soporte [13]. Sin embargo, el modelo permite un acercamiento en la predicción del FC de los combicatalizadores frente a la producción de ésteres etílicos.

Tabla 5. Valores del FC predichos según el modelo cinético para cada condición a las 24 h de reacción.

\begin{tabular}{|c|c|c|c|c|c|c|c|}
\hline & CLTC $(75: 25)$ & TLL & CALB & & CLTC (75:25) & TLL & CALB \\
\hline Condición & \multicolumn{3}{|c|}{$\begin{array}{l}\text { FC según lo obtenido por el } \\
\text { modelo }\end{array}$} & Condición & \multicolumn{3}{|c|}{ FC según lo obtenido por el modelo } \\
\hline $\begin{array}{l}\text { Efecto } \\
\text { etanol }\end{array}$ & $\%$ EE calc & $\begin{array}{c}\text { FC } \\
\text { modelo }\end{array}$ & $F C \exp$ & $\begin{array}{l}\text { Biodiésel } \\
(\%)\end{array}$ & \%EE calc & FC modelo & $F C \exp$ \\
\hline $1: 01$ & $20,05(12,73)$ & 0,63 & 0,96 & 0 & $68,49(80,11)$ & 1,17 & 1,17 \\
\hline 2:01 & $42,06(37,52)$ & 0,89 & 1,05 & 12,3 & $63,56(67,76)$ & 1,07 & 1,06 \\
\hline $3,1: 1$ & $68,49(80,11)$ & 1,17 & 1,17 & 19,2 & $44,82(65,15)$ & 1,45 & 1,33 \\
\hline $6: 01$ & $53,00(76,21)$ & 1,44 & 1,09 & 33,6 & $44,11(50,62)$ & 1,15 & 1,09 \\
\hline $\begin{array}{l}\text { Ácido graso } \\
(\%)\end{array}$ & & & & $\begin{array}{l}\text { Glicerol } \\
(\%)\end{array}$ & & & \\
\hline 1,25 & $52,25(82,80)$ & 1,58 & 1,07 & 0 & $68,49(80,11)$ & 1,17 & 1,17 \\
\hline 2,5 & $40,72(82,61)$ & 2,03 & 1,08 & 12,5 & $61,52(70,74)$ & 1,15 & 1,13 \\
\hline 3,75 & $36,48(78,27)$ & 2,15 & 1,06 & 25 & $46,80(64,39)$ & 1,38 & 1,30 \\
\hline 5 & $34,8(79,84)$ & 2,29 & 1,07 & 37,5 & $39,79(41,98)$ & 1,06 & 0,43 \\
\hline
\end{tabular}

Los valores presentados entre paréntesis corresponden a los valores obtenidos experimentalmente del \%EE.

\section{Conclusiones}

Los combi-catalizadores permiten disminuir la energía de activación aparente de la reacción de transesterificación respecto a la de los componentes mono-lipásicos que los constituyen - la del catalizador comercial Novozyme ${ }^{\circledR 435}$, esto explica en parte por qué para este sistema en particular el uso de una Combi-lipasa resulta en mayores velocidades de reacción frente a biocatalizadores comerciales.

Aunque todos los biocatalizadores estudiados fueron afectados negativamente por la presencia de glicerol añadido, el hecho de que la combilipasa de TLL $(75 \%)$ y CALB $(25 \%)$ en Lewatit $\circledast$ VP OC 1600 presente una robustez superior (y rendimientos) ante la temperatura, presencia de agua o ácidos grasos libres en el medio de reacción y nula afectación por el tipo de aceite respecto a la referencia comercial (Novozyme ${ }^{\circledR 435)}$ ), es un indicio que esta CL podría ser aplicada en la producción de biodiesel, por ejemplo, usando aceites no refinados o usados, lo que contribuye a una mayor sostenibilidad en el proceso de obtención de este biocombustible.

Los factores de complementariedad de la CL de TLL y CALB en LW para los efectos analizados, en general han mostrado sinergia en el estudio de cada uno de los efectos evaluados ( $F C>1$ ), esto da un indicio que en este Combi-catalizador la capacidad de TLL y CALB para complementarse 
va más allá de la selectividad catalítica frente a los acilglicéridos sino también frente a otros componentes (agua, glicerol, esteres etílicos) y parámetros de reacción (temperatura) en el contexto de la producción de ésteres etílicos a partir de oleína de palma y soya.

\section{Agradecimientos}

A la Universidad del Valle (Apoyo estudiantes de maestría, proyecto $\mathrm{Cl} 71139$ ) y a Colciencias (Cl 71083 - financiación 745-2016-proyecto 110671250425) por la financiación del proyecto. Agradecimientos a Lanxess (Phil Fatula) por la donación del soporte Lewatit@ VP OC 16000.

\section{Referencias bibliográficas}

[1] Ferrão-Gonzales AD, Véras IC, Silva F, Alvarez $\mathrm{HM}$, Moreau VH. Thermodynamic analysis of the kinetics reactions of the production of FAME and FAEE using Novozyme 435 as catalyst. Fuel Process Technol. 2011;92(5):1007-1011.

[2] Youngs $H$, Somerville $C$. Development of feedstocks for cellulosic biofuels. F1000 Biol Rep. 2012;4:10.

[3] Ognjanovic N, Bezbradica D, KnezevicJugovic Z. Enzymatic conversion of sunflower oil to biodiesel in a solvent-free system: Process optimization and the immobilized system stability. Bioresour. Technol. 2009;100(21):5146-54.

[4] Zhao X, Qi F, Yuan C, Du W, Liu D. Lipasecatalyzed process for biodiesel production: Enzyme immobilization, process simulation and optimization. Renew Sustain Energy Rev. 2015;44:182-97.

[5] Godoy CA, Klett J, Di Geronimo B, Hermoso JA, Guisán JM, Carrasco-López C. Disulfide Engineered Lipase to Enhance the Catalytic Activity: A Structure-Based Approach on BTL2. Int J Mol Sci. 2019;20(21):5245.

[6] Norjannah B, Ong HC, Masjuki HH, Juan JC, Chong WT. Enzymatic transesterification for biodiesel production: A comprehensive review. RSC Adv. 2016;6(65):60034-60055.

[7] Tacias-pascacio VG, Virgen-ortíz JJ, Jiménezpérez $M$, Yates $M$, Torrestiana-sanchez $B$, Rosales-quintero $A$, et al. Evaluation of different lipase biocatalysts in the production of biodiesel from used cooking oil : Critical role of the immobilization support. Fuel. 2017;200:110.
[8] Alves JS, Vieira S, Cunha AS, Silva AM, Záchia Ayub MA, Fernandez-lafuente R, et al. Combilipase for heterogeneous substrates: a new approach for hydrolysis of soybean oil using mixtures of biocatalysts. R Soc Chmistry Adv. 2014;(4):6863-6868.

[9] Poppe JK, Garcia-galan C, Matte CR, Fernandez-lafuente R, Rodrigues RC, Antônio $M$, et al. Enzymatic Optimization of synthesis of fatty acid methyl esters catalyzed by lipase $B$ from Candida antarctica immobilized on hydrophobic supports. J. Mol Catal B, Enzym. 2013;94:51-6.

[10] Khor GK, Sim JH, Kamaruddin AH, Uzir MH. Thermodynamics and inhibition studies of lipozyme TL IM in biodiesel production via enzymatic transesterification. Bioresour Technol. 2010;101(16):6558-6561

[11] Rodrigues RC, Volpato G, Wada K, Ayub MAZ. Enzymatic synthesis of biodiesel from transesterification reactions of vegetable oils and short chain alcohols.J Am Oil Chem Soc. 2008;85(10):925-930.

[12] Pedro KCNR, Parreira JM, Correia IN, Henriques CA, Langone MAP. Enzymatic biodiesel synthesis from acid oil using a lipase mixture. Quim Nova. 2018;41(3):284-291.

[13] Toro EC, Rodríguez DF, Morales N, García LM, Godoy CA. Novel Combi-lipase Systems for Fatty Acid Ethyl Esters Production. Catalysts. 2019;9(6):546.

[14] Cabrera Z, Fernandez-Lorente G, FernandezLafuente R, Palomo JM, Guisan JM. Novozym 435 displays very different selectivity compared to lipase from Candida antarctica B adsorbed on other hydrophobic supports. J Mol Catal B Enzym. 2009;57(1-4):171-176.

[15] Dallakyan S, Olson AJ. Small-molecule library screening by docking with PyRx. Methods Mol Biol. 2015;1263:243-50.

[16] Kaparthi R, Chari KS. Solubilities of vegetable oils in aqueous ethanol and ethanol-hexane mixtures. J Am Oil Chem Soc. 1959;36(2):7780.

[17] Yancy-Caballero DM, Guirardello R. Modeling and parameters fitting of chemical and phase equilibria in reactive systems for biodiesel production. Biomass and Bioenergy. 2015;81:544-555.

[18]Ávila-Cisneros N, Velasco-Lozano S, HuertaOchoa S, Córdova-López J, Gimeno M, Favela-Torres E. Production of Thermostable Lipase by Thermomyces lanuginosus on 
Solid-State Fermentation: Selective Hydrolysis of Sardine Oil. Appl Biochem Biotechnol. 2014;174(5):1859-72.

[19]Royon D, Daz M, Ellenrieder G, Locatelli S. Enzymatic production of biodiesel from cotton seed oil using t-butanol as a solvent. Bioresour Technol. 2007;98(3):648-653.

[20]Musa IA. The effects of alcohol to oil molar ratios and the type of alcohol on biodiesel production using transesterification process. Egypt J Pet. 2016;25(1):21-31.

[21] Wierschem M, Heils R, Schlimper S, Smirnova I, Górak A, Lutze P. Enzymatic Reactive Distillation for the Transesterification of Ethyl Butyrate: Model Validation and Process Analysis. Computer Aided Chem. Eng. 2015;37:2135-2140

[22] Arumugam A, Ponnusami V. Production of biodiesel by enzymatic transesterification of waste sardine oil and evaluation of its engine performance. Heliyon. 2017;3(12):e00486.

[23] Rezaei K, Jenab E, Temelli F. Effects of water on enzyme performance with an emphasis on the reactions in supercritical fluids. Critical Rev. in Biotechnology. 2007;12:183-195.

[24] Anthonsen T, Sjursnes BJ. Importance of Water Activity for Enzyme Catalysis in NonAqueous Organic Methods Non-Aqueous Enzymol. 2000:14-35. doi.org/10.1007/978-30348-8472-3_2.

[25] Chourasia VR, Gawas AS, Menon AS, Shinde PM. Production of Biodiesel by Enzymatic Transesterification using Immobilized Lipase. Heliyon. 2017;3(12):1238-1246.

[26] Ortiz C, Ferreira ML, Barbosa O, Dos Santos JCS, Rodrigues RC, Berenguer-Murcia Á, et al. Novozym 435: The "perfect" lipase immobilized biocatalyst?. Catal Sci Technol. 2019;9(10):2380-2420.

[27] Séverac E, Galy O, Turon F, Pantel CA, Condoret JS, Monsan P, et al. Selection of CalB immobilization method to be used in continuous oil transesterification: Analysis of the economical impact. Enzyme Microb Technol. 2011;48(1):61-70.

[28] Adlercreutz P. Immobilisation and application of lipases in organic media. Chem Soc Rev. 2013;42(15):6406-6436.

[29] Robles-Medina A, González-Moreno PA, Esteban-Cerdán L, Molina-Grima E.
Biocatalysis: Towards ever greener biodiesel production. Biotechnol Adv.2009;27(4):398408.

[30]Azócar L, Navia R, Beroiz L, Jeison D, Ciudad G. Enzymatic biodiesel production kinetics using co-solvent and an anhydrous medium: A strategy to improve lipase performance in a semi-continuous reactor. N Biotechnol. 2014;31(5):422-429.

[31]Aboelazayem O, Gadalla M, Saha B. Biodiesel production from waste cooking oil via supercritical methanol: Optimisation and reactor simulation. Renew Energy. 2018; 124:144-154.

[32] Lin JJ, Chen YW. Production of biodiesel by transesterification of Jatropha oil with microwave heating. J Taiwan Inst Chem Eng. 2017;75:43-50.

[33] Vieira da Silva MA, Lagnier Gil Ferreira B, da Costa Marques LG, Lamare Soares Murta A, Vasconcelos de Freitas MA. Comparative study of NOx emissions of biodiesel-diesel blends from soybean, palm and waste frying oils using methyl and ethyl transesterification routes. Fuel. 2017;194:144-56.

[34]Alejos Altamirano CA, Yokoyama L, de Medeiros JL, de Queiroz Fernandes Araújo O. Ethylic or methylic route to soybean biodiesel? Tracking environmental answers through life cycle assessment. Appl Energy. 2016;184:1246-63.

[35] Morales N, Godoy C. Lipasas Inmovilizadas Para La Producción De Biodiesel Etílico (Tesis pregrado).Cali, Colombia: Universidad del valle; 2019.

[36]Deboni TM, Hirata GAM, Shimamoto GG, Tubino M, de Almeida Meirelles AJ. Deacidification and ethyl biodiesel production from acid soybean oil using a strong anion exchange resin. Chem Eng J. 2017;333:686696.

[37] Derawi D, Abdullah BM, Zaman Huri H, Yusop RM, Salimon J, Hairunisa N, et al. Palm olein as renewable raw materials for industrial and pharmaceutical products applications: Chemical characterization and physicochemical properties studies. Adv Mater Sci Eng. 2014;2014. doi.org/10.1155/2014/134063 